\title{
Labyrinthe
}

38 | 2012 (1)

L'éloquence des singes

\section{Paroles de singes}

Anthologie assemblée et annotée par Laurent Dubreuil

\section{(2) OpenEdition}

\section{Journals}

\section{Édition électronique}

URL : http://journals.openedition.org/labyrinthe/4250

DOI : $10.4000 /$ labyrinthe.4250

ISSN : 1950-6031

\section{Éditeur}

Hermann

Édition imprimée

Date de publication : 1 avril 2012

Pagination : 103-126

ISBN : 9782705682361

Référence électronique

"Paroles de singes », Labyrinthe [En ligne], 38 | 2012 (1), mis en ligne le 01 avril 2014, consulté le 01 mai 2019. URL : http://journals.openedition.org/labyrinthe/4250; DOI : 10.4000/labyrinthe.4250

Propriété intellectuelle 


\section{Paroles de singes}

Anthologie assemblée et annotée par Laurent Dubreuil ${ }^{1}$

Le seul dénouement possible à ce dossier est une parole en archipel. Quarante-neuf extraits de soliloques, de conversations et de livres, où des singes humains parlent des autres primates et de ce qu'ils peuvent dire (ou pas), où des chimpanzés, des orangs-outangs, des bonobos, des gorilles s'expriment. Certaines citations sont fictives, évidemment, à moins qu'elles ne le soient toutes. Elles dessinent, de manière non conclusive, l'espace d'un dialogue impossible, mais ininterrompu, entre nous et nous.

L. D.

\section{[1]. Bosse-de-Nage, singe papion}

Ha ha.

Alfred Jarry, Gestes et opinions du docteur Faustroll, pataphysicien (1898), II, x, et passim.

\section{[2]. Georges-Louis Leclerc de Buffon, savant humain}

L'homme rend par un signe extérieur ce qui se passe au-dedans de lui, il communique sa pensée par la parole, ce signe est commun à toute l'espèce humaine, l'homme sauvage parle comme l'homme policé, \& tous deux parlent naturellement, \& parlent pour se faire entendre : aucun des Animaux n'a ce signe de la pensée, ce n'est pas, comme on le croit communément, faute d'organes ; la langue du singe a paru aux Anatomistes aussi parfaite que celle de l'homme: le singe parleroit donc s'il pensoit; si l'ordre de ses pensées avoit quelque chose de commun avec les nôtres, il parleroit notre langue, \& en supposant qu'il n'eût que des pensées de singe, il parleroit aux autres singes ; mais on ne les a jamais vûs s'entretenir ou discourir ensemble; ils n'ont donc pas même un ordre, une suite de pensées à leur façon, bien-loin d'en avoir de semblables aux nôtres ; il ne se passe à leur intérieur rien de suivi, rien d'ordonné, puisqu'ils n'expriment rien par des signes combinés \& arrangés ; ils n'ont donc pas la pensée, même au plus petit degré.

1. Sauf indication contraire, je traduis. 


\section{Labyrinthe, $n^{\circ} 38$}

Histoire naturelle, générale et particulière, avec la description du cabinet du roi, vol. II Histoire naturelle de l'homme, Paris, Imprimerie nationale, 1749, p. 439. L'homme est le seul singe à disposer d'un pharynx compatible avec le langage verbal. Par la suite de la mutation de certains gènes (dont FOXP2), il est également le seul primate dont les lèvres bougent de telle manière que la parole soit aisée. Quelques tentatives, limitées, de rendu vocal de mots humains chez les grands singes sont à trouver dans les citations [28], [32] et [43].

\section{[3]. César, chimpanzé leader}

Non!

Mot proféré en anglais dans Rise of the Planet of the Apes, film réalisé par Rupert Wyatt, sur un scénario de Rick Jaffa et Amanda Silver inspiré par Pierre Boulle, 2011. Cf. aussi [48] et [25] pour La Planète des singes. Kanzi (cf. [19], ainsi que l'article de Savage-Rumbaugh dans ce numéro) vocalise actuellement plusieurs dizaines de sons qui, n'entrant pas dans le répertoire usuel des bonobos, correspondent à des unités sémantiques. L'intelligibilité de ces sons distinctifs, qui semblent imiter des mots anglais dans les contraintes anatomiques données, reste fort délicate.

\section{[4]. Chantek, orang-outan de laboratoire}

NOIR ROUGE POINT [pour un groupe de poteries peintes]. YEUX BOISSON [pour du collyre]

Occurrences en langue des signes (American Sign Language, ou ASL), réalisées par Chantek et rapportées par H. Lyn Miles, responsable de l'expérience depuis 1978, dans Paola Cavalieri \& Peter Singer dir., The Great Ape Project, New York, St Martin's Griffin, 1993, p. $42-57$. Pour d'autres singes s'exprimant en langage des signes, cf. [8], [26], [35], [45].

\section{[5]. Doogie, chimpanzé narrateur}

Réveil brume, réveil brute, n'appartiens n'ici ni là.

Brouillard part, forêt noire, ni singe ni rien, qui.

Tout chat doré, mange oiseaux, mange les singes, petits. Chasse et mangouste escargot. Tous animaux.

Pour l'essentiel, les Mémoires de la jungle, de Tristan Garcia (Paris, Gallimard, 2010), sont écrits par Doogie, un singe sans doute doté de la 


\section{La grande scène des primates}

parole à la suite d'expériences scientifiques, et qui a peut-être lu Heart of Darkness ou vu Apocalypse Now.

\section{[6]. Dorothy Cheney \& Robert Seyfarth, humains enregistreurs et décodeurs}

Les vocalisations des singes vervets méritent d'être qualifiées de sémantiques, car leur fonction est de dénoter des objets et des événements dans leur environnement. Les appels des vervets sont aussi sémantiques dans le sens le plus fort du terme en ceci que leur production et leur interprétation dépendent des états mentaux de l'émetteur et du récepteur. Les vocalisations génèrent des représentations à la fois chez l'émetteur et le récepteur: les comparaisons réciproques entre vocalisations et les réponses qu'elles entraînent s'effectuent sur la base de ces représentations. [...] Bien que les représentations jouent un rôle crucial dans l'usage et l'interprétation de ces appels par les singes, nous n'avons aucune preuve que les singes (et même les grands singes) communiquent avec l'intention de changer l'état mental du récepteur ou afin d'attirer l'attention du récepteur sur le propre état mental de l'émetteur.

How Monkeys See the World, Chicago, University of Chicago Press, 1990, p. 310. Cheney et Seyfarth ont été les premiers à reprendre, avec plus de systématicité scientifique, les méthodes d'enregistrement de cris de singes mises au point par R. L. Garner (dont nous traduisons plusieurs pages dans ce dossier). Ils ont souligné l'existence, chez les vervets, de plusieurs cris d'alarmes renvoyant à des catégories de prédateurs. La citation du babouin Enzo, [13], vient du site internet des deux chercheurs. Cf. aussi [49].

\section{[7]. Cheetah, singe cinématographique, \& quelques humains}

Cheetah (décroche le téléphone).

Opératrice. Désolée, je croyais que la communication avait été établie. Un moment, s'il vous plaît.

Sam (décroche le téléphone). Allô? Allô?

Cheetah (au téléphone). Hng nh nh nh. Hououou hng

Sam. Hein? Sam à l'appareil. Qui est là ?

Cheetah. Ououp Ouououh Ouh.

Sam. Hein? J'ai dit que c'était Sam à l'appareil, voilà ce que j'ai dit.

Cheetah. Aaaaar. Aaaaar.

Sam. Qu'est-ce que vous dites? 


\section{Labyrinthe, $n^{\circ} 38$}

Cheetah. Aaaaar.

Sam. Vous n'êtes pas en train de vous payer ma tête par hasard?

Cheetah. Aaaaar. A a a a a.

Sam. N'essayez pas votre double langage avec moi, vous m'entendez?

Cheetah. Ouououououh, ouououh.

Sam. Vous... vous... vous... Grande gueule!

Cheetah. Ah ah ah ah ah ah.

Dialogue retranscrit d'après Tarzan's New York Adventure, film réalisé par Richard Thorpe en 1942, sur un scénario de Myles Connolly et al., vaguement inspiré d'Edgar Rice Burroughs. Cf. aussi [40].

\section{[8]. Nim Chimpsky, chimpanzé incompris}

Enseignante. Pomme.

Nim. Manger manger manger manger manger manger manger manger.

Enseignante. Pomme pomme manger pomme manger Nim manger pomme. [...] Tranche.

Nim. Pomme. (Nim essaie de jouer.)

Enseignante. Arrêter! (touchant la pomme) Couper.

Nim. Manger pomme.

Enseignante. Pomme. Couper pomme couper.

Nim. Manger pomme (main gauche). Nim (main droite). [L'enseignante tape la main gauche de Nim pour l'interrompre.] Manger (avec la main droite).

Enseignante (après avoir fait un geste indéchiffrable avec ses deux mains). Arrêter! Assez!

Nim. Manger.

Nim Chimpsky fut ironiquement nommé en référence à Chomsky luimême (cf. [9]) par Herbert Terrace, qui, n'arrivant pas à démontrer sa première hypothèse sur les capacités langagières des grands singes, déclara qu'aucun primate autre que les humains ne pouvait acquérir la langue des signes. Cf. l'ouvrage de Terrace, Nim, New York, Columbia University Press, 1987 [2éd.]. Certains extraits des vidéos sont disponibles à l'URL http://kanzi.bvu.edu, accompagnés de transcriptions et commentaires par Sue Savage-Rumbaugh. Pour d'autres singes s'exprimant en langage des signes, cf. [4], [26], [35], [45].

\section{[9]. Noam Chomsky, Homo linguisticus}

On peut apprendre à des humains comment produire une imitation correcte du complexe système de communication des abeilles. Cela n'est 


\section{La grande scène des primates}

d'aucune espèce d'intérêt pour les entomologistes, qui sont rationnels, et qui comprennent quelque chose à la science [...]. Il est évidemment impossible d'obtenir des financements afin d'enseigner à des doctorants comment devenir des abeilles imparfaites. [...] Dans le même ordre d'idées, il est possible qu'entraîner des singes à faire quelque chose de signes puisse nous éclairer un peu sur les capacités cognitives des singes. [...] Pauvre Nim. [...] Résultat final de l'expérience? Exactement ce qu'un biologiste sain d'esprit aurait supposé: zéro. Vient ici le plus triste. Les chimpanzés peuvent devenir assez violents lorsqu'ils vieillissent, du coup les scientifiques étaient prêts à envoyer Nim au paradis des singes. Mais les expérimentateurs étaient tombés amoureux de lui, et ils firent tous les efforts pour le sauver. Il a finalement été envoyé dans une sorte de ferme pour les chimpanzés, où, j'imagine, il mourut en paix — priant le Seigneur en langage des signes.

Extrait d'un échange par courrier électronique avec Matt Aames Cucchiaro en 2007 et 2008. L'intégralité de l'entretien écrit se trouve à l'URL: http://www.chomsky.info/interviews/2007----.htm. Cf. [8] pour Nim Chimpsky.

\section{[10]. Hélène Cixous, primate poète}

Qu'est-ce qu'un Jeune Saint Juif? S'agissant de Jacques Derrida, l'inventeur de la différance, le poète de la danse de l'écrit avec l'ouïe, ouïe si souvent inouie, on aura bien entendu, par homophonie, que ce portrait est en douce celui d'un jeune sainjuif, je veux dire un singe juif, s'il y en a, et pourquoi n'y aurait-il pas de singe saint ou de saint singe?

«Prière d'insérer » du Portrait de Jacques Derrida en Jeune Saint Juif, Paris, Galilée, 2001.

\section{[11]. Daniel Dennett, homme d'idées}

$Q$. Donc vous pensez que si je disais à Kanzi en langage des signes [sic] Viens, raisonnons ensemble, il ne se passerait pas grand-chose?

Dennett. Je pense que c'est comme parler à Dieu. Vous pouvez toujours dire cela à Kanzi, mais Kanzi ne va pas vous répondre.

Entretien mené par Roger Bingham pour The Science Studio, 2 novembre 2007, http://www.thesciencenetwork.org. Sur Kanzi, [19] et [29]. 


\section{Labyrinthe, $n^{\circ} 38$}

\section{[12]. Birago Diop, griot humain}

Bouki a raconté la mésaventure de M'Bile-la-Biche à Golo-le-Singe . Golo a colporté l'histoire. Toute la brousse l'a sue.

Les Contes d'Amadou Koumba, Paris, Présence Africaine, 1947, fin de

« Tours de lièvre ». Comme le dit Diop dans « Maman-Caïman », Golo

le singe est le griot des animaux de la brousse.

\section{[13]. Enzo, babouin des savanes}

Ouaaah-hou. [...] Ouah.

Retranscrit dans la phonétique du français d'après l'enregistrement sonore disponible à l'adresse http://www.sas.upenn.edu/ seyfarth/ Baboon\%20research/wahoos.htm. Les «wahoos» chez les babouins (à la différence des «wows » américains) sont surtout utilisés pour signaler une menace particulière ou signifier une position sociale. Ils sont alors modulés différemment. Chaque individu a par ailleurs une intonation spécifique, qui n'est pas fixe tout au long de la vie. Les deux «wahoos » de la citation sont prononcés différemment en fonction de leurs places (initiale et finale) dans une séquence de cris.

\section{[14]. François Froger, humain voyageur}

Les Singes y sont plus gros \& méchans qu'en aucun endroit de l'Afrique; les Negres les craignent, \& ils ne peuvent aller seuls dans la campagne sans courir risque d'estre attaquez de ces Animaux, qui leur présentent un bâton, \& les obligent à se battre. J'ay entendu dire aux Portugais, que souvent ils les avoient vû porter sur les arbres de petites filles de 7 à 8 ans, \& qu'on avoit une peine incroyable à les leur oster. La pluspart des Negres croyent que c'est une Nation étrangère, qui s'est venuë peupler dans leur Pais, \& qu'ils ne parlent point de peur de travailler.

Relation d'un voyage fait en 1695, 1696, \& 1697, aux côtes d'Afrique, Amsterdam, Schelte, 1699, p. 43. Les singes en question (habitant la Guinée portugaise) sont sans doute des chimpanzés. Cf. [18] et [41].

\section{[15]. Gustave Flaubert, humain adolescent et halluciné}

«Que me voulez-vous?», dit-elle avec effroi aussitôt qu'elle l'eût reconnu.

Et il s'ensuivit un long silence. Il ne répondit pas et fixa sur elle un regard dévorant. Puis, se rapprochant de plus en plus, il prit sa taille de 


\section{La grande scène des primates}

ses deux mains et déposa sur son cou un baiser brûlant qui sembla pincer Adèle comme la morsure d'un serpent. Il vit sa chair rougir et palpiter.

«Oh! je vais appeler au secours », s'écria-t-elle avec effroi. « $\mathrm{Au}$ secours! au secours! Oh! le monstre! » ajouta-t-elle en le regardant.

Djalioh ne répondit pas. Seulement il bégaya et frappa sa tête avec colère. Quoi ! ne pouvoir lui dire un mot ! ne pouvoir énumérer ses tortures et ses douleurs, et n'avoir à lui offrir que les larmes d'un animal et les soupirs d'un monstre! Et puis être repoussé comme un reptile! Être haï de ce qu'on aime et sentir devant soi l'impossibilité de rien dire! Être maudit et ne pouvoir blasphémer!

"Quidquid volueris» (1837), ch. IX. Djalioh est le fils d'une «négresse » et du «plus bel orang-outang qu'on eût jamais vu» (cf. ch. V). Djalioh, dont le public ignore la nature hybride, vit avec son gardien, Paul, et tombe amoureux de la jeune femme de celui-ci (Adèle). Il la viole et la tue, après avoir assassiné la progéniture du couple, et avant de se suicider d'un bond fatal, « la tête la première sur la cheminée de marbre » (ch. IX).

\section{[16]. Gille, singe exemplaire}

[...] Venez de grace,

Venez Messieurs : Je fais cent tours de passe-passe.

Cette diversité dont on vous parle tant,

Mon voisin Leopard l'a sur soi seulement:

Moy je l'ay dans l'esprit: vostre serviteur Gille,

Cousin \& gendre de Bertrand,

Singe du Pape en son vivant,

Tout fraîchement en cette ville

Arrive en trois basteaux, exprés pour vous parler:

Car il parle, on l'entend, il sçait danser, baler,

Faire des tours de toute sorte,

Passer en des cerceaux ; \& le tout pour six blancs :

Non Messieurs, pour un sou: si vous n'êtes contens,

Nous rendrons à chacun son argent à la porte.

Jean de La Fontaine, «Le Singe et le Léopard », Fables choisies, vol. IV, Paris, Thierry \& Bardin, 1679, p. 18-19; boniment du singe. 


\section{Labyrinthe, $n^{\circ} 38$}

\section{[17]. Ishmaël, gorille romanesque}

Dans l'espace de quelques années, je devins capable de suivre la plupart des conversations qui parvenaient à mes oreilles [...]. Je savais désormais que j'étais un gorille et que Zaza [dans la roulotte à la droite de la mienne] était un chimpanzé. Je savais aussi que tous les habitants des roulottes étaient des animaux.

Daniel Quinn, Ishmael, New York, Bantam, 1992, p. 15. Le narrateur du roman de Quinn relate comment il fut l'élève privé d'Ishmaël.

\section{[18]. Richard Jobson, humain esclavagiste}

[Les Babouins] sont assurément une race et un type de peuple qui, afin de ne pas être contraints de travailler et de vivre dans la servitude, refusent de parler.

The Golden Trade, Londres, Okes, 1623, p. 153. Ces « Babouins » en sont peut-être; il s'agit en tout cas de primates africains. Cf. [14] et [41].

\section{[19]. Kanzi, bonobo génial \& Sue Savage-Rumbaugh, humaine à proportion}

Kanzi est dans une pièce close. Une porte vitrée donne sur la « salle collective » où se trouve (entre autres) Matata, mère de Kanzi. Sue Savage-Rumbaugh est derrière une autre vitre et parle ainsi à Kanzi.

Sue. Bonjour. Comment ça va Kanzi ? Est-ce que tu voulais quelque chose?

Kanzi va vers la porte et jappe en montrant la serrure. Il crie et va taper contre le mur opposé.

Sue. Je n'aime pas ça.

Kanzi (va vers le clavier, dans un autre coin de la pièce, tout en jappant légèrement; puis presse une touche sur le clavier, enclenchant la voix synthétique). CLES. CLES.

Sue. Tu as besoin de la clé? (Kanzi jappe. Il regarde en direction de Sue et tend la main droite.) Tu veux la clé, hein? J'ai des clés ici.

Kanzi (tout en jappant). SALLE COLLECTIVE CLES.

Sue. Tu veux ouvrir, hein?

K. jappe $[\ldots]$

Kanzi. CLES MATATA GENTIL.

Sue. Oh, tu veux voir Matata et tu vas être gentil. (Kanzi jappe de manière répétée, avec excitation). Je suis contente d'entendre cela, c'est très bien. (Kanzi se précipite vers la porte.) 


\section{La grande scène des primates}

Transcrit d'après l'enregistrement vidéo, inclus dans le documentaire télévisé Kanzi: An Ape of Genius (1993), NHK. L'expérience de Sue Savage-Rumbaugh avec une famille bonobo est largement décrite au sein de ce numéro dans l'article coécrit par la scientifique, et dans le texte de Laurent Dubreuil. Voir quelques mots de Panbanisha, sœur de Kanzi, [29]; ainsi que d'autres occurrences avec des lexigrammes dans les citations [21] et [39].

\section{[20]. Julien Offray de La Mettrie, philosophe visionnaire}

Parmi les Animaux, les uns apprennent à parler \& à chanter; ils retiennent des airs et prennent tous les tons aussi exactement qu'un Musicien. Les autres, qui montrent cependant plus d'esprit, tels que le Singe, n'en peuvent venir à bout. Pourquoi cela, si ce n'est par un vice des organes de la parole?

Mais ce vice est-il tellement de conformation, qu'on n'y puisse apporter aucun remède? En un mot, seroit-il absolument impossible d'apprendre une Langue à cet Animal? Je ne le croi pas.

Je prendrois le grand Singe préférablement à tout autre, jusqu'à ce que le hazard nous eût fait découvrir quelqu'autre espèce plus semblable à la nôtre, car rien ne répugne qu'il y en ait dans des Régions qui nous sont inconnuës. [...] Je le prendrois aux mêmes conditions des Écoliers d'Amman ${ }^{2}$; c'est-à-dire que je voudrois qu'il ne fût ni trop jeune, ni trop vieux ; car ceux qu'on nous aporte en Europe sont communement trop âgés. Je choisirois celui qui auroit la physionomie la plus spirituelle, \& qui tiendroit le mieux dans mille petites opérations, ce qu'elle m'auroit promis. Enfin, ne me trouvant pas digne d'être son Gouverneur, je le mettrois à l'École de l'excellent Maître que je viens de nommer, ou d'un autre aussi habile, s'il en est.

L'Homme Machine, Leyde, Luzac, 1748, p. 26sq. Cf. aussi [27], [31], [38] et [47] pour un rapprochement comparable entre grands singes et humains sourds-muets.

\section{[21]. Lana, chimpanzée volubile, \& Tim, expérimentateur humain}

Timothy Gill (Tim) met du chou dans le tourniquet, à la place de la boisson que Lana avait d'abord demandée. Tim et Lana communiquent

2. Il s'agit de Johann Conrad Amman, qui mit au point une méthode pour enseigner aux sourds-muets à parler, d'abord exposée dans Surdus loquens, Amsterdam, Wettstein, 1692. 
par l'intermédiaire d'une machine, en pressant des touches portant des lexigrammes.

Lana. S'IL TE PLAIT MACHINE DONNER MORCEAU DE CHOU (bis)

Tim.? QUOI DANS MACHINE

Lana. TIM BOISSON

Tim.? QUOI DANS MACHINE

Lana. TIM TOURNER

Tim.? QUOI DANS MACHINE

Lana. TIM METTRE CHOU DANS MACHINE

Duane Rumbaugh dir., Language Learning by a Chimpanzee, New York, Academic Press, 1977. Dans cette expérience, dirigée par Rumbaugh, Lana devait apprendre des formules toutes faites et contraignantes (comme « S'IL TE PLAIT MACHINE DONNER $X \gg)$, afin d'obtenir des résultats avec ses expérimentateurs. L'évaluation portait sur les rapports logiques et syntaxiques, au moins autant que sur le sémantisme et l'aspect perlocutoire de la communication. Pour d'autres singes utilisant des lexigrammes: [19], [29], [39].

\section{[22]. André Leroi-Gourhan, humain paléoanthropologue}

Les deux formes de mémoire technique se trouvent, pour des motifs différents, comme assurés de leur conservation hors de l'individu luimême, la mémoire instinctive lui apportant un comportement hérité de l'espèce et la mémoire d'éducation lui offrant une somme de connaissances possédées par le groupe social. [...] La mémoire de l'homme est coulée dans le langage, elle est totalement socialisée et constitue un capital de pratiques transmissible d'une génération à l'autre. [...] La parole est un outil verbal, isolable de la bouche qui l'émet comme l'outil manuel est isolable de la main. [...] Extérieure à l'individu, la mémoire sociale totalise d'une génération sur l'autre les innovations individuelles, ce qui n'est le cas pour aucune des formes de la mémoire animale.

"Technique et société chez l'animal et chez l'homme », in Recherches et débats du centre catholique des intellectuels français, $n^{\circ} 8,1957$, p. 23-25. 


\section{[23]. Linné, humain taxinomiste}

Homo nocturnus [...]. Homo sylvestris Orang Outang. [...] Corps blanc, marche debout [...] Espérance de vie, 25 ans. Le Jour est aveugle, se cache; la Nuit voit, sort, chaparde. Il parle en sifflant; Il pense, réfléchit.

Systema naturae, $12^{\mathrm{e}}$ édition, 1766, vol. I, p. 33. Linné, qui s'attira ensuite les railleries de Buffon (lire [2]), semblait considérer les orangs-outangs (et sans doute les chimpanzés) comme une espèce d'hommes troglodytes et nocturnes, capables de communication par une langue sifflée.

\section{[24]. Marmot, singe fantasque}

Dequoy donc te plains-tu, quand mesme en cet affaire

Il ne se peut trouuer de trauail plus leger.

[D'après Marcus Gheeraerts aîné,] « D'un Singe et d'un petit chat », Esbatiment moral des animaux, Anvers, Galle, [1587], p. 63. La fable d'origine est l'une des premières versions rimées du récit sur le singe usant d'un chat (et plus précisément de ses pattes) afin de tirer les marrons du feu.

\section{[25]. Ulysse Mérou, humain du cosmos, et autres singes}

Je me rappelais maintenant une discussion [...] avec un spécialiste. Il m'avait appris que de graves savants passaient une partie de leur existence à essayer de faire parler des primates. Ils prétendaient que rien dans la conformation de ces bêtes ne s'y opposait. Jusqu'alors, tous leurs efforts avaient été vains, mais ils persévéraient, soutenant que le seul obstacle tenait à ce que les singes ne voulaient pas parler. Peut-être, un jour avaient-ils voulu, sur la planète Soror? [...]

Il me faut maintenant confesser que je m'adaptai avec une aisance remarquable aux conditions de vie dans ma cage. Au point de vue matériel, je vivais dans une félicité parfaite: dans la journée, les singes étaient aux petits soins pour moi; la nuit, je partageais la litière d'une des plus belles filles du cosmos. [...]

Écoutez-moi, ô singes! car je parle; et non pas, je vous l'assure, comme une mécanique ou un perroquet. Je pense, et je parle, et je comprends aussi bien ce que vous dites que ce j'énonce moi-même.

Extraits du manuscrit rédigé (en français ou en « langage simien ») par Ulysse Mérou et laissé dans l'espace, in Pierre Boulle, La 


\section{Labyrinthe, $n^{\circ} 38$}

Planète des singes, Paris, Julliard, 1963; I, XI; II, I; III, vIII. Cf. deux extraits d'adaptations filmiques: [3] et [48].

\section{[26]. Michael, gorille à signes}

ÉCRASE VIANDE GORILLE. BOUCHE DENT. PLEURE BRUIT-AIGU FORT. MÉCHANT PENSE ENNUIS VISAGE-VOIS. COU-COUPÉ LÈVRES [FILLE] TROU.

Michael (1973-2000) était, avec la femelle Koko, l'un des deux gorilles à utiliser le langage des signes (ASL). D'après Francine Patterson, qui est l'enseignante de ces deux primates, les mots ci-dessus (cités d'après la transcription anglaise qu'elle a donnée) correspondent à une description, par Michael, du récit de l'assassinat de sa mère par des braconniers dans la jungle. (Dans le dialecte de Koko (adopté par Michael), LEVRES renvoie à fille, femme, d'où la notation entre crochets.) Vidéo disponible à l'URL: http://www.koko.org/world/ kokoflix.php?date $=2008-03-23$. Pour d'autres singes s'exprimant en langage des signes, cf. [4], [8], [35], [45].

\section{[27]. James Burnett Monboddo, philosophe pithécophile}

Si donc le langage n'est pas essentiel à la nature des hommes, ni si aisé à acquérir qu'il résulterait de l'opération naturelle d'une mère élevant son enfant; il suit, par conséquence, qu'il y eut un temps où les hommes ne parlaient pas. Or je désire savoir, dans ces conditions, ce que serait le critère, la marque distinctive d'une différence entre les hommes et les Orangs-outangs dans leur présent état? Et même, je vais aller plus loin; je désire qu'un philosophe me dise la différence spécifique entre un Orang-outang assis à table, et se comportant ainsi que M. de La Brosse ou M. de Buffon l'ont décrit, et un sourd et muet; et, en général, je crois qu'il sera très difficile, ou plutôt impossible, pour un homme accoutumé à faire des divisions entre les choses sur la foi de marques spécifiques et non sur celles de différences individuelles, de tracer une ligne démarquant les Orangs-outangs de ceux d'entre nous qui sont sourds et muets. Ils ont dans les deux cas des organes permettant la prononciation, et, dans les deux cas, montrent des signes d'intelligence par leurs actions, avec la différence, sans doute, que les sourds, parce qu'ils sont éduqués parmi des hommes civilisés, ont plus d'intelligence. Mais comment est-il possible que, de cette différence de plus ou de moins seulement, et qui s'explique si bien, on les conçoive comme étant de différentes espèces? 


\section{La grande scène des primates}

Ainsi me suis-je efforcé de soutenir l'ancienne définition de l'homme, et de montrer qu'y entre l'Orang-outang, quoiqu'il n'ait point d'usage du langage. [...] Supposons qu'à force d'études, il doive être découvert que les Orangs-Outangs n'ont pas seulement inventé l'art de construire des huttes, et d'attaquer ou se défendre à l'aide de bâtons, mais ont aussi trouvé une manière de communiquer avec les absents, et d'enregistrer leurs idées par la méthode de la peinture ou du dessin, comme cela se pratique dans de nombreuses nations barbares (et la supposition n'est pas du tout impossible, ni même improbable); et supposons qu'ils se soient dotés de quelque forme de gouvernement, et élisent des rois ou des souverains, ce qui est possible [...], devraient-ils être toujours comptés parmi les brutes, parce qu'ils n'ont pas encore découvert la méthode pour communiquer par des sons articulés?

[...] Dotés d'une intelligence humaine, et semblablement des organes pour la prononciation, ils doivent nécessairement posséder la capacité de l'apprentissage, par enseignement et imitation, s'ils n'ont pas celle d'inventer un langage ; et qui a la capacité d'apprendre à parler, cela suffit à le nommer un homme, quand même il n'atteint pas au plein exercice de cette faculté ; puisque la nature humaine, comme nous l'avons observé ailleurs, consiste essentiellement en des capacités. [... Cette] expérience n'a jamais été tentée rigoureusement sur aucun Orang-outang jusqu'ici apporté en Europe. Car il ne semble point qu'aucun soin ait jamais été pris pour apprendre à l'un d'eux à parler. Nous ne pouvons donc pas affirmer qu'ils n'apprendraient pas cet art, si les mêmes soins leur étaient prodigués qu'aux élèves de M. Braidwood ${ }^{3}$.

Of the Origin of Language and Progress, Édimbourg, Balfour, 1774, vol. I, p. 297-300. Cf. aussi [20], [31], [38] et [47] pour un rapprochement comparable entre grands singes et humains sourds-muets.

\section{[28]. L'Orang-outang anonyme, primate loquace}

Papa! Papa! Papa! - Cup! Cup! Cup!

L'explorateur et conservateur William Furness III travaillait dans les années 1909 et suivantes sur la cognition des primates avec Lightner Witmer, qui apparaitt plus bas dans cette anthologie, [32]. Furness rapporte que ces deux mots (souvent prononcés par rafales) représentaient

3. Thomas Braidwood (1715-1806), éducateur écossais, qui, la même année que l'abbé de l'Épée en France (1760), décida d'instruire les sourds-muets. 


\section{Labyrinthe, $n^{\circ} 38$}

l'étendue du vocabulaire anglais oral d'une femelle orang-outang, après enseignement. Selon l'auteur, ces mots étaient proférés avec une intention sémantique et référentielle; cf. ses «Observations on the mentality of chimpanzees and orang-utans ", Proceedings of the American Philosophical Society, LV, 1916, p. 283sq. Cf. aussi [43] pour une autre tentative comparable.

\section{[29]. Panbanisha, bonobo facétieuse}

BUNNY toi.

À l'automne 2010, Panbanisha participe à une plaisanterie, et - au moyen d'une combinaison entre lexigramme et geste déictique informe le journaliste de CNN Anderson Cooper qu'il va devoir endosser le rôle de Bunny. Panbanisha et son demi-frère Kanzi ([19]) sont deux bonobos, éduqués par Sue Savage-Rumbaugh et ses collaborateurs. Dans les années 1980, Savage-Rumbaugh avait créé les personnages de Bunny (un gentil lapin) et de Gorilla (un gorille malfaisant) comme éléments de référence normatifs pour les singes. La vidéo est disponible à l'URL http://www.youtube.com/watch?v-qsUHuurFLXM, et ailleurs. Pour d'autres singes utilisant des lexigrammes: [21], [29], [39].

\section{[30]. Panzee, chimpanzé vocale}

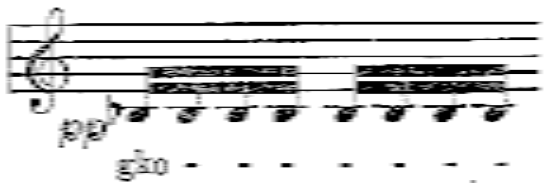

Transcription par Blanche Learned dans l'ouvrage cosigné avec Robert Yerkes, Chimpanzee Intelligence and its Vocal Expression, Baltimore, Williams \& Wilkins, 1925, p. 120. Selon Learned, gko (émis avec une imbrication du $g$ et du $k$ «sans équivalent en anglais ») était utilisé par Panzee en forme de salutation. L'auteur souligne que le cri est en général répété, et que le tempo varie selon les situations, tous éléments qui sont conformes aux études plus contemporaines de la communication vocale chez les primates (cf. [6] et [49]). 


\section{[31]. Samuel Pepys, humain si curieux}

Appelé chez Sir W. Batten pour voir l'étrange créature que le Capitaine Holmes a rapporté de Guinée avec lui ; c'est un grand babouin, mais comme un homme pour la plupart, et à tel point que, même si l'on dit qu'il en existe toute une espèce, je ne puis croire que j'eus affaire à autre chose qu'un monstre descendant d'un homme et d'une femelle babouin. Je crois qu'il comprend déjà assez l'anglais, et je suis d'avis qu'on peut lui enseigner à parler ou faire des signes.

Extrait du Diary, à la date du 24 septembre 1661. Le «monstre » en question n'est vraisemblablement ni un hybride, ni un babouin, mais un chimpanzé. Cf. aussi [20], [27], [38] et [47] pour un rapprochement comparable.

\section{[32]. Peter, chimpanzé de théâtre \& Lightner Witmer, psychologue pionnier}

Peter. Mama!

Witmer. Si un enfant privé de la parole m'était confié et que, dès le premier essai, il parvienne à articuler le son $p$ aussi promptement que l'a fait Peter, j'en viendrais à penser que cet enfant est à même d'apprendre la plupart des éléments du langage articulé dans l'espace de six mois. Les résultats du test d'écriture, au cours duquel la capacité d'imitation de Peter lui a permis de former la lettre $\mathrm{W}$, autorisent à penser que Peter pourrait apprendre à écrire quelques mots au moins au bout d'un an d'instruction. Il ne saurait y avoir de doute que, dans une certaine mesure, il comprend déjà le langage parlé.

Extraits de Witmer, «A Monkey with a mind », The Psychological Clinic III-7, 1909. Peter, élevé dès l'enfance par les époux McArdle, se produisait sur des scènes de théâtre en habit noir. Mama était le seul mot qu'il articulait alors. C'est lors d'une tournée du singe à Philadelphie que Witmer, professeur de psychologie à l'Université de Pennsylvanie, fait connaissance avec Peter et le soumet à de nombreux tests d'intelligence. Ce même Peter est sans doute l'inspiration de Kafka pour son récit « Ein Bericht für eine Akademie », cf. [37]. Pour d'autres mots humains prononcés par des singes, cf. aussi [28] et [43].

\section{[33]. Claude Perrault, humain naturaliste}

Les Muscles de l'Os Hyoïde, de la Langue, du Larynx, et du Phraynx, qui servent la pluspart à articuler la parole, estoient entierement semblables 
à ceux de l'Homme, \& beaucoup plus que ceux de la Main; dont néanmoins le Singe, qui ne parle point, se sert presque avec autant de perfection que l'Homme: ce qui fait voir que la parole est une action plus particulière à l'Homme, \& qui le distingue davantage des Brutes que la Main qu'Anaxagore, Aristote, \& Galien ont estimé estre l'organe que la Nature a donné à l'Homme comme au plus sage de tous les Animaux, peut-estre faute d'avoir fait cette réflexion.

Suite des mémoires pour servir à l'histoire naturelle des Animaux, Paris, Imprimerie nationale, 1671, p. 126. Ces remarques découlent des observations permises par la dissection de deux sapajous et de deux « guenons » (cercopithèques).

\section{[34]. Melchior de Polignac, large cardinal, à l'Orang-outang du Roi}

Parle, et je te baptise.

Telle est la phrase que Bordeu (dans la Suite du Rêve de D'Alembert, de Denis Diderot, fin du dialogue rédigé en 1782) attribue au cardinal de Polignac. Ce propos s'adresse à l'« orang-outang qui a l'air d'un SaintJean qui prêche au désert [...] au Jardin du Roi, sous une cage de verre. »

\section{[35]. Rinnie, orang-outang instruite}

TOI PLUS RIZ. - TOI DONNER.

Exemples d'énoncés formulés par Rinnie, orang-outang dans une réserve naturelle d'Indonésie, après quatre mois d'enseignement du langage des signes par Gary Shapiro et Biruté Galdikas. Cité d'après Coll., The Mentalities of Gorillas and Orangutans, Cambridge (G. B.), Cambridge University Press, 1999, ch. 13. Pour d'autres singes s'exprimant en langage des signes, cf. [4], [8], [26], [45].

\section{[36]. Clément Rosset, vieux singe philosophe}

Si les Chimpanzés ne parlent guère notre langue, du moins la comprennent-ils passablement; au lieu que nous, qui sommes déjà incapables de reproduire correctement les sons dont ils usent, n'avons pas été plus heureux, malgré notre belle science, dans nos tentatives de comprendre jamais un traître mot de chimpanzé. [...] Il est bien certain que le Chimpanzé n'a jamais fait de latin. Mais ce qu'il faudrait savoir, c'est pourquoi le Chimpanzé n'a pas eu accès à la culture latine. Lui en a-t-on donné les possibilités ? L'occasion? Le goût? [...] Mettez en cage 


\section{La grande scène des primates}

un de nos jeunes enfants, si doué soit-il, nourrissez-le exclusivement de cacahuètes, et il ne vous récitera pas Tite-Live.

Lettre sur les chimpanzés, Paris, Gallimard, 1965, p. 35-37. Dans cet ouvrage d'une ironie à la fois glaçante et bien informée, Rosset tourne obliquement en dérision les mouvements politiques d'émancipation (dont le féminisme). Curieusement, nombre d'arguments avancés par Rosset dans un but de dénonciation pourraient être aujourd'hui repris tels quels par des militants de la cause animale.

\section{[37]. Rotpeter, singe kafkaïen, \& un narrateur humain}

"Quand, en face à face, je vous entends parler, Rotpeter, que je vous vois boire, vraiment - qu'il s'agisse là pour vous d'un compliment ou pas, c'est tout de même la vérité - j'en oublie tout à fait que vous êtes un chimpanzé. Ce n'est que peu à peu, en sortant de mes pensées et en retournant vers la réalité, que mes yeux me rappellent de qui je suis l'invité.

«Oui. »

$[\ldots]$ « Puisque nous avons déjà commencé,j'ose la question: depuis combien de temps êtes-vous tout à fait parmi nous?»

« Cinq ans, le cinq août cela fera cinq ans. »

« Voilà qui est inouï. En cinq ans, abjurer l'état de singe et galoper à travers toute l'évolution humaine. Vraiment, personne ne l'a encore fait. Sur cette piste, vous êtes tout seul.»

« Je sais que c'est beaucoup et parfois cela dépasse même mon entendement. Mais à d'autres heures plus calmes, je ne juge pas que cela soit si merveilleux. »

Esquisses préparatoires de Franz Kafka pour son récit ultérieur «Ein Bericht für die Akademie », in Nachgelassene Schriften und Fragmente, vol. I, 1993, p. 385sqq. Le "Rapport à une académie» contient le seul texte du chimpanzé, délesté de toute intervention humaine. Il fut d'abord publié par Martin Buber dans son journal Der Jude (1917), puis par Kafka dans le volume Ein Landartzt (1920). Il a été suggéré4 que Rotpeter (Pierre le Rouge) avait été inspiré par un chimpanzé de musichall, nommé Peter (qui se produisit à Prague en $1908^{5}$ ). J'ajoute que ce même singe est sans doute celui qui fut aussi à l'origine de la recherche expérimentale de Furness et Witmer; cf. [28] et [32].

4. Entre autres par Peter-André Alt, Franz Kafka, Munich, Beck, 2005, p. 522.

5. Cf. Prager Tagblatt, 17.09.1908, p. 5. 


\section{[38]. Marquise de Saint-Ferdinand, femme philosophe}

Si l'on abandonnoit un enfant à sa vivacité; si on lui permettoit de sauter, de courir, de grimper, d'aller chercher sa nourriture dans les bois; après sept ou huit ans de ce genre de vie, auroit-il plus d'esprit, seroit-il plus méditatif, plus propre à saisir de hautes spéculations que ne l'est un vieux singe? Retournons cette expérience, saisissons-en le revers, puisque nos enfans seroient comme les singes si on les abandonnoit à leur mobilité capricieuse; le singe pourroit profiter, comme nos enfans, de l'éducation, \& devenir un Philosophe si l'on avoit assez d'industrie pour fixer sa mobilité, pour lui apprendre à commercer d'idées avec nous, à nous communiquer les siennes, à saisir les nôtres. Sa langue n'est pas assez bien organisée pour parler; eh bien l'instituteur l'enseigneroit, comme on enseigne les muets de naissance; on conviendroit de signes avec cet animal; on pourroit lui apprendre à lire et à écrire, comme on l'apprend à un sourd de naissance. Rien ne me révolte tant quand je pense que l'homme, que le Philosophe ne pense point à multiplier les êtres pensans.

Joseph-Adrien Lelarge de Lignac, Examen sérieux et comique des Discours sur l'esprit, vol. II, Amsterdam, [s.n.], 1759, p. 31-32. Sera ensuite invité à l'appui un orang-outang savant qui s'avérera, quelques centaines de pages plus loin, être en fait un mulâtre posant au singe. La marquise, instruite par l'épisode, abjurera la philosophie. Cf. aussi [20], [27] et [47] pour un rapprochement comparable entre grands singes et humains sourds-muets; cf. aussi [31].

\section{[39]. Sherman \& Austin, chimpanzés malins}

Sherman. CAROTTE STEVE. - DONNER LAIT LIQUIDE. SHERMAN CHATOUILLER ROYCE. - TELE PEUR. Austin. AUSTIN DONNER CAFE CHAUD. - AUSTIN DONNER PECHE. - SHERMAN DONNER CACAHUETE. - OUI PRENDRE AUSTIN VITAMINE.

Exemples d'énoncés (non consécutifs), réalisés par les chimpanzés Austin et Sherman à l'aide d'un clavier électronique figurant des lexigrammes. Certaines occurrences reproduisent des séquences produites précédemment par les chercheurs; d'autres s'en inspirent; d'autres encore n'ont pas d'antécédent dans la discussion. Citations d'après Sue Savage-Rumbaugh, Ape Language, New York, Columbia University Press, 1986, p. 286-298. Pour d'autres singes utilisant des lexigrammes: [19], [21], [29]. 


\section{La grande scène des primates}

\section{[40]. Tarzan, homme-singe}

« Je suis Tarzan, » cria-t-il, « je suis un grand tueur. Que tous respectent Tarzan des Singes et Kala, sa mère. Il n'y a personne parmi vous qui soit aussi puissant que Tarzan. Gare à ses ennemis. » [...] « Ma mère était un Singe; bien sûr, elle ne pouvait pas m'en dire beaucoup plus. »

Edgar Rice Burroughs, Tarzan of the Apes, Londres, Methuen, 1917, p. 68 et 256.

\section{[41]. Garcilaso de la Vega, humain mémorialiste}

Puisque [les singes] se comprennent les uns les autres en criant (je soutiens pour ma part que tous les animaux et oiseaux font ainsi avec les membres de leur espèce), les Indiens disent qu'ils savent parler et qu'ils dissimulent leurs paroles aux Espagnols, de peur que ceux-ci ne les envoient dans les mines d'or ou d'argent.

Comentarios reales de los Incas (1609-1617), VIII, XVIII. L'auteur, surnommé «l'Inca », est considéré comme le premier écrivain métisse de langue espagnole. De nombreuses espèces de primates vivaient et vivent encore au Pérou, dont les capucins, mais l'homme est le seul grand singe à hanter cette partie du globe. Cf. [14] et [18].

\section{[42]. Valmiki, auteur épique}

Le héros né de Raghou dit au fils du Vent sur le point de partir luimême : "Hanoûmat, prince des singes, je ne t'ai pas récompensé comme il faut. Choisis donc une grâce; car le service que tu m'as rendu est bien grand. » À ces mots, des larmes de joie troublant ses yeux, celui-ci dit à Râma: "Que mon âme reste jointe à mon corps, sire, aussi longtemps qu'il sera parlé de Râma sur la terre; je demande cette grâce, si tu veux m'en accorder. »

À peine eut-il articulé ces mots que Râma lui fit cette réponse : «Qu'il en soit ainsi! La félicité descende sur toi! Jouis de la vie, sans maladie, sans vieillesse, toujours vigoureux et jeune, aussi longtemps que la terre soutiendra les mers et les montagnes! »

La Mithilienne alors de lui faire aussi une grâce nonpareille: «Que les différentes choses à manger, fils de Mâroute, se présentent d'elles-mêmes à toi sur la terre! Que les chœurs des Apsaras, les Gandharvas, les Dânavas et les Dieux t'honorent comme un Immortel en tous lieux où tu seras. Que partout il naisse pour l'amour de toi ou ruisselle à ton gré, quadrumane sans péché, des fruits pareils à l'ambroisie et des ondes limpides! » 
« Ainsi soit-il ! r reprit le singe, qui partit les yeux mouillés de larmes; et tous ses compagnons de s'en aller, comme ils étaient venus, à leurs différentes habitations, s'entretenant tout le voyage, tant ils aimaient Râma, des grandes aventures de ce noble Raghouide.

Le Ramayana, adapté par Hippolyte Fauche, Paris, Librairie internationale, 1864, vol. II, p. 331-332. Il s'agit de la version réduite, rédigée dans ce français biblico-homérique, qui fit tant pour l'intérêt de Michelet et d'autres. Hanuman est le protagoniste du cinquième livre du Ramayana, le «Sundara Kanda », où il retrouve la trace de Sita, la promise de Rama. L'extrait que je cite ici décrit un moment de la cérémonie d'intronisation de Rama, qui apparaît dans le sixième livre du récit («Yuddha Kanda »).

\section{[43]. Viki, chimpanzé parlant}

Mama.

«Mama» est l'un des quelques mots que Viki pouvait articuler de manière signifiante en contexte, dans l'expérience menée au cours des années 1940 et 50 par Keith et Catherine Hayes; cf., par exemple, leur article "The intellectual development of a home-raised chimpanzé », Proceedings of the American Philosophical Society, XCV-2, avril 1951, p. 107sq. Pour d'autres mots humains prononcés par des singes, cf. aussi [8] et [32].

\section{[44]. Karl Christoph Vogt, humain méthodiquement délirant}

Le singe $[\ldots]$ cultive l'imitation des mouvements, pour la figure comme pour le corps, refusée aux oiseaux ; il n'essaie au contraire presque jamais ou dans une limite très-restreinte, comme le fait l'oiseau, de contrefaire le ton, la modulation et l'articulation des mots, qu'il entend. [...] Sophie est sous ce point de vue complètement singe. [...] Il n'y a pas de trace de langage articulé ou même de son entente. [...] Le seul mot à demi articulé que Sophie a appris à l'hospice est: Amen! encore le prononce-t-elle très mal et à moitié seulement; le A est poussé avec une forte aspiration, et l'M est plutôt un éternuement étouffé. C'est à cela que se bornent ici les manifestations d'une «âme parlante ». Sophie Wiss est donc singe par les facultés intellectuelles, par l'épine dorsale, femme prognathe de race inférieure par la figure, de race blanche par le corps.

Mémoire sur les microcéphales ou hommes-singes, vol. XI des Mémoires de l'institut national genevois, Genève, Georg, 1867, p. 169sq. Vogt, à 


\section{La grande scène des primates}

partir d'une interprétation libre de Darwin, pensait que, par leur développement limité, les microcéphales représentaient « des phases antérieures du développement du genre humain » (p. 199), et qu'ils donnaient une image de ce qu'étaient les « hommes-singes » de la Préhistoire.

\section{[45]. Washoe, chimpanzé artiste ROUGE CHAUD ÉLECTRIQUE}

Titre donné par Washoe en langage des signes à l'une de ses peintures (cité par Roger Fouts \& Stephen Tukel Mills, Next of Kin, New York, Morrow, 1997, p. 280). Washoe (1965-2007), d'abord éduquée par les époux Gardner, est le premier grand singe à avoir atteint un niveau important de communication par la langue des signes (ASL). Réformée de la NASA, où elle participait aux préparations des voyages spatiaux, Washoe a joué un rôle absolument déterminant dans la mise en place des recherches modernes sur l'acquisition du langage humain par des primates. Pour d'autres singes s'exprimant en langage des signes, cf. [4], [8], [26], [35].

\section{[46]. Wu Cheng'en, interprète du Singe-Roi}

Le Beau Singe-Roi avait joui des générosités de la nature depuis trois, quatre siècles, cinq cents ans, peut-être, quand un beau jour, au cours d'un joyeux banquet, il se sentit soudain accablé de tristesse et se mit à verser des larmes. Les singes alarmés lui demandèrent en le saluant bien bas:

«- Pourquoi Votre Majesté se tourmente-t-Elle?

- J'ai beau vivre présentement dans la joie, je ne peux me défendre de penser un tout petit peu plus loin: c'est ce qui me tourmente. [...] Certes, nous n'avons aujourd'hui ni à nous soumettre aux lois d'un roi humain, ni à redouter les menaces des animaux, mais plus tard, quand viendront la vieillesse et la décrépitude, ne tomberons-nous pas aux mains de ce vieux bonhomme de Yama, au royaume des ombres? À l'instant où périra notre corps, n'aurons-nous pas vainement vécu en ce monde, si nous ne pouvons demeurer indéfiniment parmi les êtres divins?»

À ce discours chaque singe se couvrit le visage et se mit à sangloter pitoyablement tant ils étaient tous accablés par la pensée de l'impermanence. Voilà que soudain saute hors des rangs un gibbon au long dos qui, d'une voix forte, interpelle le roi:

«-Qu'une telle pensée ait assailli Votre Majesté signifie en vérité que votre esprit commence à s'ouvrir à la Voie. Parmi les cinq sortes de 


\section{Labyrinthe, $n^{\circ} 38$}

créatures, il n'existe que trois catégories d'êtres qui ne soient pas soumis à la juridiction du roi des Enfers.

- Lesquelles, le sais-tu?

- Celles de bouddha, d'immortel et de saint, qui échappent au cycle de la transmigration, qui ne naissent ni ne meurent et jouissent de la même longévité que le ciel et la terre, ses montagnes et ses rivières.

- Où habitent ces trois-là?

- En ce monde-ci, dans de vieilles grottes et dans les montagnes d'immortels. »

Ces explications remplirent d'aise le roi des singes. Il déclara: « Je vous ferai mes adieux dès demain et descendrai de la montagne. Quand bien même il me faudrait aller comme le nuage jusqu'aux trois extrémités du ciel ou de la mer, j'en trouverai de ces trois-là pour apprendre à rester jeune et vivre indéfiniment, afin d'échapper à jamais à l'embuscade que nous tend seigneur Yama!»

La Pérégrination vers l'Ouest (Xijou ji), I, 1. Traduction française André Lévy, Paris, Gallimard, 1991, vol. I. Cf. aussi le personnage d'Hanuman, dans le Ramayana; [42].

\section{[47]. Robert Yerkes, grand primatologue}

Les grands singes parlent-ils? Tout semble indiquer que leurs vocalisations ne constituent pas un vrai langage [...]. Apparemment, les sons expriment principalement des émotions innées. Cela surprend, au regard des preuves de l'existence d'idées, ou, occasionnellement, d'actions procédant de l'intuition d'une solution [insight]. Nous ne pouvons supposer avec beaucoup d'assurance qu'ils n'ont rien d'autre à exprimer que des sentiments, ni même que leurs sons ressemblant à des mots manquent de signification idéationnelle. Peut-être que la raison principale à l'incapacité parmi les singes de développer la parole tient à l'absence d'une tendance à l'imitation sonore. La vision stimule fortement en eux l'imitation; mais l'ouïe ne semble pas avoir un tel effet. Je suis incité à conclure de ces divers éléments que les grands singes ont beaucoup à dire, mais aucun talent pour la manipulation de sons représentant des sentiments ou des idées individuelles (en contraste avec celles de leur race). Peut-être peut-on leur enseigner à utiliser leurs doigts, un peu à la façon d'une personne sourde et muette, et les aider ainsi à acquérir un simple et non vocal « langage des signes». 


\section{La grande scène des primates}

Almost Human, New York, The Century $C^{\circ}$, 1925, fin du ch. 9, p. 179sq. Robert Yerkes, qui fut professeur de psychologie à Harvard et Yale, fonda dans cette dernière université un laboratoire d'études des primates, avant d'organiser une station expérimentale autonome à Orange Park, en Floride. Cette dernière structure fut ensuite transférée à Emory University, où de nombreuses recherches essentielles sur la cognition des primates ont pris place depuis les années 1970 (avec les Rumbaugh, M. Tomasello, F. De Waal). Cf. aussi [20], [27], et [38] pour un rapprochement comparable entre grands singes et humains sourds-muets; cf. encore [31].

\section{[48]. Zira, chimpanzé psychologue \& Zaius, orang-outan gardien du temple}

Zira. Regardez [l'astronaute humain Taylor]! Il bouge ses doigts.

Zaius. Parce qu'il a vu que vous bougiez les vôtres.

Zira. Peut-être qu'il a compris.

Zaius. Un humain n'a pas de compréhension. On peut lui apprendre à faire quelques simples tours. Rien de plus.

Planet of the Apes, film réalisé par Franklin Schaffner en 1968, sur un scénario de Rod Serling et Michael Wilson, d'après le roman de Pierre Boulle. Cf. aussi [3] et [25].

\section{[49]. Klaus Zuberbühler, humain à oreilles}

Des combinaisons particulières de cris sont données en réponse à un nombre d'événements à la fois externes et discrets, comme la chute d'un arbre ou d'une grosse branche, les cris d'alarme d'un groupe voisin au loin, ou un prédateur éloigné. La part commune de ces différents contextes tient à l'absence de danger immédiat, au contraires des situations où les émetteurs sont surpris par la proximité d'un prédateur. Or, quand ils entendent une combinaison de cris d'alarmes introduites par un boum, les singes Diane ne répondent pas avec leurs propres cris d'alarme, en net contraste avec leur réponse vocale aux cris normaux - c'est-à-dire dénués d'un boum introducteur - des singes des palétuviers.

« Linguistic prerequisites in the primate lineage », in Maggie Tallerman dir., Language Origins, Oxford, Oxford University Press, 2005, p. 277. Klaus Zuberbühler a été le doctorant de Cheney et Seyfarth, cités plus haut dans cette anthologie (cf. [6] et [13]). Il va plus loin que ses anciens directeurs dans l'interprétation des vocalisations par des singes 


\section{Labyrinthe, $n^{\circ} 38$}

et déchiffre, en plus du régime sémantique, une syntaxe des appels. Il suppose aussi des formes d'intelligibilité dans la communication orale entre primates d'espèces différentes mais vivant ensemble. 